\title{
Analysis of Signal Reception Mechanism of Sky Wave Long-distance Maritime Communication
}

\author{
Huantao Ren ${ }^{1,}$, Qiangjun $\mathrm{Xie}^{2}$, Jiajing Chai ${ }^{3}$ and $\mathrm{Yi} \mathrm{Xue}^{4}$ \\ ${ }^{1}$ College of Electronics and Information, Hangzhou Dianzi University, 310018, Hangzhou, China \\ ${ }^{2}$ School of Sciences, Hangzhou Dianzi University, 310018, Hangzhou, China \\ ${ }^{3}$ School of Computer Science and Technology, Hangzhou Dianzi University, 310018, Hangzhou, China \\ ${ }^{4}$ College of Automation, Hangzhou Dianzi University, 310018, Hangzhou, China
}

\begin{abstract}
Based on the principle of high frequency radio propagation, the signal reception mechanism of sky wave communication over oceans is investigated. Due to long distance signal transmission, the energy loss is inevitable, especially in the space and on the sea surface. Firstly, we establish the space propagation loss model by the ionospheric absorption, the free space propagation characteristics and other extra loss. Referring to the reflection principle of smooth ground and the Kirchhoff approximation, the energy loss models of the calm sea surface and the turbulent sea surface are obtained respectively. Then, through combining the space propagation loss model and the sea surface propagation loss models, we give out a formula of receiving point field strength. According to the signal to noise ratio, we summarize a complete and concise sky wave maritime communication calculation process, through which multi hops number of the receivable sky wave signal can be calculated accurately. The experimental results show the effectiveness.
\end{abstract}

\section{Introduction}

As shown in Fig.1, multi-hop high frequency(HF, defined to be $3-30 \mathrm{MHz}$ ) radio wave propagation is a main method applied to the field of telecommunications. The HF waves travel by multiple reflections off the ionosphere(the Flayer) and off the earth just like the 'multi hops'. This propagating method has many valuable advantages, such as low cost, non-destructive ionosphere of relay system. It is widely used in the researches of the long-distance maritime communications(see [1-3] and the references therein)

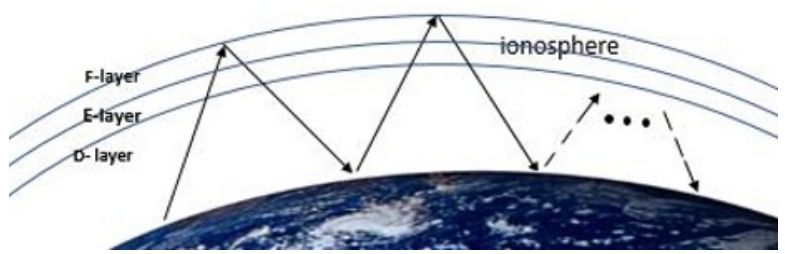

Figure 1: Diagram of the multi-hop HF wave propagation

The maritime propagation process comprises with free space propagation, ionospheric reflection and absorption, sea surface absorption and scattering. The affecting factors of ionospheric wave propagation, such as usable frequency, absorbing attenuation and sunspot number, are extremely complicated with time and frequency. The propagation of waves on the sea surface varies with the frequency and the state of the sea. The turbulence on the sea surface can affect the scattering of radio waves. In 2003, Anderson [1] developed a computational model which could compute the contributions of diffusely-scattered fields arising from non-specular reflection at the ground reflection points for multiple-hop propagation paths. In 2008, Kong et al [3] proposed an aggregated-path routing approaching for inter-ship communications in wireless multi-hop networks. Tian and $\mathrm{Lu} \mathrm{[4]} \mathrm{analyzed} \mathrm{the} \mathrm{relationship} \mathrm{between} \mathrm{radio}$ wave frequency and sky wave propagation path. In 2014, Sherstyukov et al [5] investigated the change of E-layer parameter on the base of experimental values. However, few literatures investigate the complete signal reception mechanism of sky wave multi-hop propagation.

In this paper, the whole signal reception mechanism of the martitime communication is consided, the complete energy loss model is established and the value of multi hops of the receivable skywaves is calculated.

The paper is organized as follows. Section 2 introduces the sky-wave propagation mode, process and propagation conditions with diffrent emission angles. In Section 3, the sky-wave space propagation is divided into a free space propagation and an ionosphere propagation, some energy loss formulas are analyzed. In Section 4, sea surface propagation models are obtained. The ocean situation is divided into calm ocean and turbulent ocean. By Kirchhoff approximation, the scattering coefficients are obtained respectly. In Section 5, using the result of space propagation loss, ionosphere loss as well as absorption and scattering of the sea surface, a complete process loss model is achieved. In Section 6, introducing a concept of signal to noise ratio (SNR), multi hops numbers of

a Corresponding author: huantao119@foxmail.com 
propagation on the calm and the turbulent sea surface respectively. Section 7 presents the conclusion.

\section{Condition and process of HF radio wave propagation over oceans}

Because of differences in the emission angle of the radio waves, the propagating paths in free space are different, which means energy loss is veried.

\subsection{Condition of high-frequency radio wave reflection in the ionosphere}

The ionospheric conditions are constantly changing as the solar radiation intensity is always altering. For example, after sunrise or in summer, the sun's rays will increase, the concentration of electrons in each ionosphere layer will change, and sunspots will move more violently. In general, the high frequency waves will be absorbed in the D and E layers and be reflected back in the $\mathrm{F}$ layer.

When the elevation angle is $\theta$, the highest frequency of ionospheric energy reflection is

$$
f_{\max }=\sqrt{\frac{80.8 N_{\max }\left(1+\frac{2 h}{R}\right)}{\sin ^{2} \theta+\frac{2 h}{R}}}
$$

Here, $N_{\max }$ is the maximum electron density of the ionosphere, $h$ is the distance from the Earth's surface to the ionosphere, and $R$ is the radius of the Earth. By (1), the highest frequency that the ionosphere can reflect at different elevation angles can be calculated as Table 1 .

Table 1. The highest frequency and elevation angle of the ionospheric energy reflection table

\begin{tabular}{|c|c|c|c|c|c|}
\hline$\theta /$ degree & 0 & 30 & 45 & 60 & 90 \\
\hline$f_{\max } / \mathrm{MHz}$ & 30.6 & 16 & 12.2 & 10.2 & 8.9 \\
\hline
\end{tabular}

As shown in Table 1, the highest frequency range that the ionosphere can reflect is $8.9 \mathrm{MHz}$ to $30.6 \mathrm{MHz}$, and short-wave frequency range is $3-30 \mathrm{MHz}$, so it can be concluded that the short-wave can be reflected regardless of the elevation angle.

\subsection{High-frequency radio wave propagation mode}

In the process of radio propagation, transmission loss is affacted by many factors, the basic transmission loss of radio in the transmission process $L_{b}$ can be divided into four parts [6], the expression is as follow:

$$
L_{b}=L_{b f}+L_{a}+L_{g}+Y_{p},
$$

where, $L_{b f}$ is propagation loss of atmosphere, $L_{a}$ is ionospheric absorption loss, $L_{g}$ is ground emission loss, $Y_{p}$ is additional loss, the units of each index are expressed in $\mathrm{dB}$. Let $L_{g}=0$ for the maritime propagation. The whole process is shown in Figure 2.

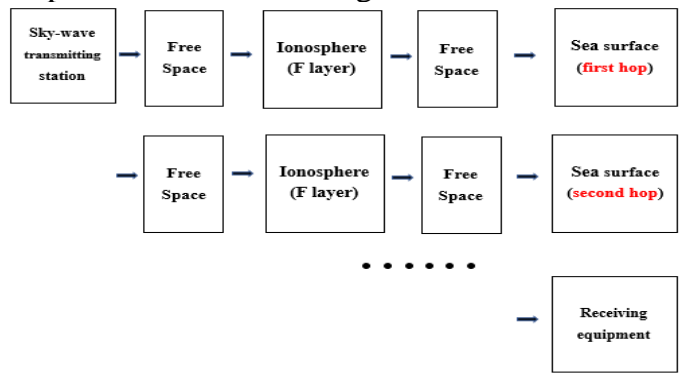

Figure 2 Diagram of HF radio wave propagation mode

\section{Space propagation loss}

In this paper, we divide the space wave propagation loss into free space propagation loss, ionospheric propagation loss and extra loss, and analyze these processes separately.

\subsection{Free Space Propagation Loss}

Free space transmission loss is a phenomenon in which the energy of the wave naturally diffuses increasingly as the propagation distance increases. The farther the propagation distance is, the larger the loss is, which is the main component of the transmission loss of the sky wave. The free space proposed here is an ideal situation, that is to say, the medium is uniform, conductivity $\sigma=0$, relative dielectric constant $\varepsilon_{r}=1$, relative magnetic permeability $\mu_{r}=1$.

The definition of free space transmission loss is: when direction coefficients of the transmitting antenna and the receiving antenna are both 1 , the ratio of the transmitting antenna's radiated power $\mathrm{P}_{\mathrm{r}}$ to the receiving antenna's optimal receiving power $\mathrm{P}_{\mathrm{L}}$ is recorded as $\mathrm{L}_{\mathrm{bf}}$, the equation is:

$$
L_{b f}=10 \cdot \lg \frac{P_{r}}{P_{L}}
$$

It can be known from the theory of antenna, when the receiving antenna polarization and impedance are matching [6], the receiving power $P_{\mathrm{r}}$ (Watt)

$$
P_{r}=S A_{e}=\frac{P_{t} G_{t}}{4 \pi d^{2}} \cdot \frac{\lambda^{2} G_{r}}{4 \pi}=\left(\frac{\lambda}{4 \pi d}\right)^{2} P_{t} G_{t} G_{r},
$$

where, $S$ is Poynting vector, $A_{e}$ is effective area of receiving antenna, $P_{t}$ is input power of a transmitting antenna, $G_{t}$ and $G_{r}$ are the gains of the transmitting antenna and the receiving antenna respectively. $\lambda$ is the wave length of the sky wave, $G_{t}$ is emitting antenna gain, $d$ is the distance.

By (4), the free space propagation loss is

$$
L_{b f}=\left(\frac{4 \pi d}{\lambda}\right)^{2}
$$


which is expressed in decibels $(\mathrm{dB})$ as

$$
L_{b f}(d B)=32.45+20 \log _{10} f(M H z)+20 \log _{10} d_{e f f}(\mathrm{~km}) .
$$

Here $f$ is working frequency, $d_{\text {eff }}$ is effective propagation distance, the equation of $d_{\text {eff }}$ is as follows[7]:

$$
d_{e f f}=2 n a_{0} \frac{\sin \left(D / 2 n a_{0}\right)}{\cos \left(\Delta+D / 2 n a_{0}\right)},
$$

where, $D$ is arc length from the transmitter to the receiver, $\mathrm{n}$ is hop-count of sky-wave propagation, $a_{0}$ is earth radius $(6730 \mathrm{~km}), \Delta$ is directivity angle of a transmitting antenna, we can calculate formula(7) through the following formula:

$$
\Delta=\arctan \left[\cot \frac{D}{2 n a_{0}}-\frac{a_{0}}{a_{0}+H} \csc \frac{D}{2 n a_{0}}\right],
$$

where, $H$ is effective height of the ionosphere. For instance, we suppose the effective height of the E layer is a constant $H=110 \mathrm{~km}$.

\section{2 lonospheric absorption loss}

The ionospheric absorption loss is second factor in the short-wave propagation loss. For shortwaves, it mainly absorbed by the $\mathrm{D}$ and the $\mathrm{E}$ layer when the electric wave passes through the ionosphere. The ionospheric absorption loss is also related to the number of sunspots, solar zenith angle, working frequency, radiation elevation angle, and magnetic frequency. It can be calculated from the following semi-empirical formula[7]

$$
\sec i_{100}=\frac{1}{\cos \left(\frac{\pi}{2}-\frac{D}{2 \mathrm{a}_{0}}-\beta\right)},
$$

with

$$
\beta=\operatorname{arctg}\left[\left(\cos \frac{D}{2 a_{0}}-\frac{a_{0}}{a_{0}+110}\right) \sin \left(\frac{D}{2 a_{0}}\right)\right],
$$

where, $I$ is absorption index, the expression of absorption index $I$ is as follows:

$$
I=(1+0.037 R)(\cos 0.881 \chi)^{1.3},
$$

where, $R$ is the mean value of sunspot variation for 12 months, and by referring to Sunspot Index and Long-term Solar Observations (Silso) website [8], we can know the value of $\mathrm{R}$ is 21.7. $\chi$ means the solar zenith angle of two absorbing lands at $100 \mathrm{~km}$. Moreover, in this paper, we mainly consider ionospheric absorption loss of $\mathrm{D}$ and $\mathrm{E}$ layer, because the two layers absorb much energy. The loss is only in the daytime. At night, the electron density of the $\mathrm{D}$ and $\mathrm{E}$ layer is very thin, the absorption loss is so small that it could be ignored (when $\chi \geq 102.2^{\circ}$, the index $I$ will not exist).

Because D layer absorbs the most energy, and most radio cannot arrive $\mathrm{F}$ layer, so absorption mainly happens on E layer. According to the Rec. ITU-R P.1240-1 [9], we can know that the maximum usable frequency (MUF) prediction value for the $\mathrm{E}$ layer is:

$$
\mathrm{MUF}_{E}=3.94+2.80 x-1.70 x^{2}-0.60 x^{3}+0.96 x^{4},
$$

where, $x$ is

$$
x=\frac{D-1150}{1150}
$$

Moreover, the MUF of E layer is $28 \mathrm{~dB}$ [10], so we can calculate $D$ value through formula (12) and (13).

\subsection{Extra loss}

The definition of $Y_{p}$ is other loss except for above indicators, it includes polarization loss of electric wave, polar loss and defocus effect of the ionosphere, and so on. That is a comprehensive estimate. $Y_{p}$ relates to local time of the reflection point [7], and the loss values are shown in Table 2:

Table 2. Check list of additional loss and time

\begin{tabular}{cc}
\hline Time & Additional loss $Y_{p}(\mathrm{~dB})$ \\
\hline $22 \sim 04$ & 18 \\
$04 \sim 10$ & 16.6 \\
$10 \sim 16$ & 15.4 \\
$16 \sim 22$ & 16.6 \\
\hline
\end{tabular}

Because the ionospheric absorption loss is apparent in the daytime, so we take value $15.4 \mathrm{~dB}$ (the corresponding time is 10 16) as calculated value in the following experiments.

\section{Ocean surface reflection strength model}

When radio waves arrived at the surface of the sea, scattering will happen on the surface, so we need to calculate the scattering coefficient, but it will change with the sea surface turbulence. Thus, we divide oceans into two conditions, calm ocean and turbulent ocean, wherein, the calm sea surface can be considered as sea surface without scattering and the turbulent sea can scatter radio wave.

\subsection{Calculation of the reflection coefficient for the calm ocean surface}

According to Snell law, which is a reflection law of represent plane wave slants to the interface of an ideal medium [11], we can obtain Fresnel reflection coefficient formula for horizontal and vertical polarization waves of the calm ocean surface:

$$
\begin{aligned}
& R_{H}=\frac{\sin -\sqrt{\varepsilon-\cos ^{2} \theta}}{\sin \theta+\sqrt{\varepsilon-\cos ^{2} \theta}}, \\
& R_{V}=\frac{\varepsilon \sin \theta-\sqrt{\varepsilon-\cos ^{2} \theta}}{\varepsilon \sin \theta+\sqrt{\varepsilon-\cos ^{2} \theta}},
\end{aligned}
$$


where, $\theta$ is grazing incidence angle, by using the value of $\tilde{\varepsilon}$, we can get the value of horizontally polarized wave $\left(R_{H}\right)$ and vertical polarization wave $\left(R_{V}\right)$.

Referring to the expression of ground reflection loss [5] because the process of ground reflection and ocean reflection are similar, we obtain the expression of calm ocean reflection absorption loss $L_{m}$ is

$$
L_{m}=10 \cdot \log _{10}\left(\frac{R_{v}^{2}+R_{h}^{2}}{2}\right)(n-1) .
$$

The amount of energy loss multiplied by reflection losses value is the total amount of reflection loss, that is to say, when the hop number of reflections is (n-1).

Considering $L_{m}$, we can obtain the field strength after reflection $E_{0}$, the expression of reflection strength of the calm ocean is

$$
E_{0}=137.2+20 \log _{10} f+G_{t}+P_{t}-L_{b}-L_{m}(d B \mu V / m) .
$$

\subsection{Calculation of the reflection coefficient for the turbulent ocean surface}

\subsubsection{The reflection of the turbulent ocean surface}

For the turbulent ocean reflection, we use a modifying factor to approximate the reflection coefficient of turbulent ocean surface on the basis of calm ocean surface reflection coefficient. The equation is:

$$
R^{\prime}=\rho R,
$$

where, $R^{\prime}$ is reflection coefficient of turbulent ocean, $\rho$ is modifying factor, $\mathrm{R}$ is calm ocean surface reflection coefficient. We can obtain the expression of modifying factor given by CCIR [12] as following:

$$
\rho=\frac{1}{\sqrt{3.2 g-2+\sqrt{(3.2 g)^{2}-7 g+9}}},
$$

with

$$
g=0.5\left(\frac{4 \pi \delta f \sin \theta}{c}\right)^{2}
$$

where, $c$ is light speed, $\mathrm{f}$ is frequency of radio wave, $\mathrm{h}$ is root-mean-square height of ocean surface, and $\delta$ can be gain according to the Phillips wave model:

$$
\delta=0.0053 U^{2},
$$

where, $U$ represents wind speed near the sea level, whose unit is $\mathrm{m} / \mathrm{s}$. Using formula (19), (20) and (21) to get $\rho$, and then reflection coefficient of turbulent ocean $R^{\prime}$ can be obtained through formula (18).

\subsubsection{Approximate method of sea surface scattering}

Kirchhoff approximation (KA) method is suitable for scattering calculation of turbulent surface, which is a similar concept of tangent plane, that is to say, KA method using reflection field on a point to replace the scattering field.

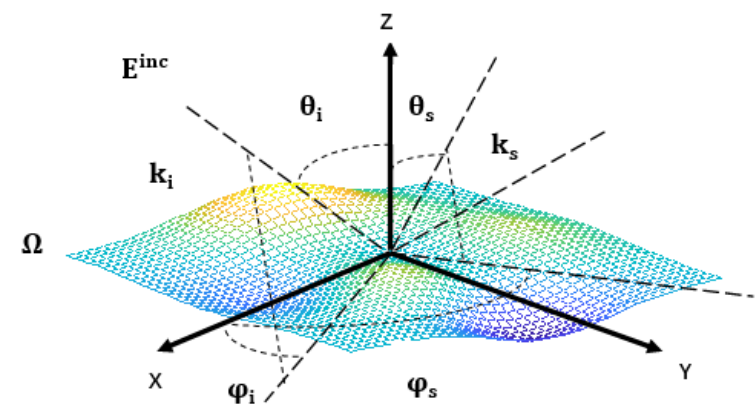

Figure 3. Kirchhoff scattering diagram of turbulent ocean

According to the definition of scattering coefficient, we can obtain the expression of the scattering coefficient $\left(\sigma_{p q}\right)$ by this approximation method:

$$
\sigma_{p q}=\frac{\left(k_{1} q\left|U_{p q}\right|\right)^{2}}{2\left|q_{z}\right|^{4} h^{2}\left|\rho^{\prime \prime}(0)\right|} \exp \left[-\frac{\left(q_{x}^{2}+q_{y}^{2}\right)}{2 q_{z}^{2} h^{2}\left|\rho^{\prime \prime}(0)\right|}\right]
$$

where, $k_{1}=\frac{2 \pi}{\lambda} \sqrt{\varepsilon_{r}}, \quad \varepsilon_{r}=70, h=0.0051 U^{2}, \rho(0)$ is the surface correlation function.

\section{Modelling}

Through the loss model of each subprocess obtained in the previous section, we can connect them together and find the total energy lost during the propagation.

When the ocean reflection strength $E_{0}$ and atmosphere noise strength $E_{n}$ are known, we can obtain the reception signal-to-noise ratio SNR:

$$
S N R=E_{0}-E_{n}(d B \mu V / m),
$$

where, $E_{0}$ is reflection strength of calm ocean, $E_{n}$ is the atmosphere noise strength.

According to the electromagnetic field theory, we can know the relation between SNR and power $\mathrm{P}$ is as following:

$$
P=10^{\frac{S N R}{10}} \text {. }
$$

Referring to the formula (2) and (6), we can obtain field strength of the receiving point with unit $(\mathrm{dB} \cdot \mu \mathrm{V} / \mathrm{m})$ as following [7]:

$$
E=137.2+20 \log _{10} f+G_{t}+P_{t}-L_{b}(d B \mu V / m),
$$

According to all above formulas and descriptions, we can summarize the important and basic equations for the calm ocean surface and the turbulent ocean surface.

\subsection{HF radio wave propagation loss model of the calm ocean}


For the calm ocean surface, we can get the formulas as following:

$$
\left\{\begin{aligned}
E_{0}= & 137.2+20 \log _{10} f+G_{t}+P_{t}-L_{b}- \\
& L_{m}(n-1)(d B \mu V / m) \\
L_{m}= & 10 \cdot \log _{10}\left(\frac{R_{v}{ }^{2}+R_{h}^{2}}{2}\right) \\
E_{h}= & \sigma_{p q} E
\end{aligned}\right.
$$

\subsection{HF radio wave propagation loss model of the turbulent ocean}

For the turbulent ocean surface, we can obtain following formulas:

$$
\left\{\begin{aligned}
E_{0}^{\prime}= & 137.2+20 \log _{10} f+G_{t}+P_{t}-L_{b}- \\
& \left(L_{m}^{\prime}+E_{h}\right)(n-1)(d B \mu V / m) \\
L_{m}^{\prime}= & 10 \cdot \log _{10}\left(\frac{\left(\rho R_{v}\right)^{2}+\left(\rho R_{h}\right)^{2}}{2}\right) \\
E_{h}= & \sigma_{p q} E
\end{aligned}\right.
$$

where, $E_{h}$ is scattering loss intensity, $E_{0}^{\prime}$ is reflection strength of the turbulent ocean, $L_{m}^{\prime}$ is the turbulent ocean reflection absorption, $\sigma_{p q}$ is scattering coefficient.

\section{Experimental results of the model}

Through the series of formulas in the upper sections, we can get the different hop number between the calm ocean surface and the turbulent ocean surface.

Here, we set the initial sky wave signal transmitting power is 100 watt.

Atmospheric noise is the main source of noise in maritime communication, which is mainly caused by lightning. Because noise is affected by the ionosphere, and the strength of noise will alter with the frequency, season, geographical position and climate. Generally, the intensity of atmospheric noise will decrease with the increase of frequency. The atmospheric radio noise intensity formula [6] is:

$$
E_{n}=-0.0019 f^{4}+0.098 f^{3}-1.8 f^{2}+13 f-13(d B),
$$

where, $f$ is working frequency.

As we all know, the targets cannot be detected by the sensor when SNR below $10 \mathrm{~dB}$, so we get the SNB evaluation criteria

$$
S N R=E_{0}-E_{n}>10(d B)
$$

By (28), SNB's formula in (23) and the other formulas of the upper chapters, we can get the SNB attenuation curves on the calm ocean and the turbulent ocean surface. The experimental results are shown in Figure 4:

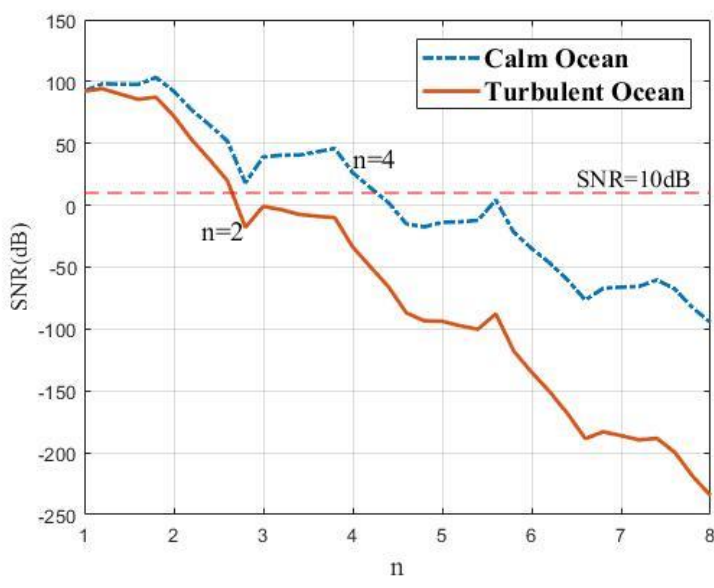

Figure 4. The signal to noise ratio(SNB) attenuations of the calm ocean and the turbulent ocean surface

According to the curves in Fig.4, we know that the maximum hop number of the calm ocean is 4 , and the maximum hop number of the turbulent ocean is 2 . The result shows that this mechanism is reliable. That is, the experimental result is effective to the investigation of the multi hops of the HF sky wave maritime communication.

\section{Conclusions}

In this paper, the signal reception mechanisms of the HF sky wave communication over oceans are theoretically analyzed. Through the ionospheric absorption principle, reflection principle of sea surface and the Kirchhoff approximation, energy loss models of the sky wave signal reception on the calm sea surface and the turbulent sea surface are obtained, respectively. The maximum sky wave multi-hop numbers of the signal receiving can be given by the SNB attenuation curves. The results reflect rationality of the analysis on maritime communication signal reception mechanism.

\section{References}

1. S. Anderson. IEEE Radar Conf, 497-503 (2003)

2. L.Q. Yang, J.M. Fan, L.X Guo, W Liu, X Lee, J Feng, $\mathrm{P}$ Lou, Z.X Lu. IEEE Trans. Antennas Propag, 66,4821-4831 (2018)

3. P.Y. Kong, M.T. Chou, J.S.Pathmasuntharam. ICT, 89-94 (2008)

4. X.P. Tian, J.H. Hu. 2009 CiSE 1-4 (2009)

5. O.N. Sherstyukov, A.D. Akchurin, R. Latipov. URSI GASS. 1-2 (2014)

6. X.B. Yuan. Chinese J. of information and communications. 5, 11-12 (2013)

7. G.C Qu, R. Wang. Ship Electronic Engineering, 29, 92-98 (2009)

8. http://www.sidc.be/silso/yearlyssnplot. Yearly mean and monthly smoothed sunspot number.

9. Basic maximum usable frequency (MUF), Actual MUF, and Ray Prediction Method, ITU-R P.1240-1 (1997-2007) 
10. Yannick Gerini. Shortwave propagation and communications systems, (2012)

11. Y. Wang,J. Gu. Electronic Design Engineering, 24. 113-116(2016)

12. CCIR: Computer program GRWAVE 